\title{
Supporting Information: Enhanced Molecular Diffusivity Through Destructive Interference Between Electrostatic and Osmotic Forces
}

Tuhin Samanta, ${ }^{1}$ Setare Mostajabi Sarhangi, ${ }^{1}$ and Dmitry V. Matyushov ${ }^{1,}$ a)

School of Molecular Sciences and Department of Physics, Arizona State University, PO Box 871504, Tempe, AZ 85287-1504

\section{SI. SIMULATION PROTOCOL}

Large scale Atomic/Molecular Massively Parallel Simulator (LAMMPS) MD package is used for the production of trajectories. MD integration followed the standard protocol with the integration time-step of $1 \mathrm{fs}$, the NVT ensemble, and the SPC/E force field for water as is done in a number of previous simulation studies of solvation $^{1}$ and mobility ${ }^{2-4}$ in water.

Three masses are placed to the spherical solute with the radius $R=3 \AA$ : mass $m_{c}=120$ a.u. at the geometrical center and two small masses $m=30$ a.u. on both sides from the center at the distance of $R / 2$. The total mass of the solute is $M=180$ a.u., which translates to the thermal velocity $v_{T}=\sqrt{k_{\mathrm{B}} T / m}=117 \mathrm{~m} / \mathrm{s}, T=300$ $\mathrm{K}$. Charge $q$ is located at one of masses $m$, at the distance $R / 2$ from the geometric center (Figure $\mathrm{S} 1$ ). This protocol allows us to create a configuration in which only electrostatic torque contributes to the rotations of the solute. The torque from the Lennard-Jones (LJ) potential is zero since the LJ force is applied to the center of mass. The solute-water LJ potential is defined by Lorentz-Berthelot rules $^{5}$ with $\sigma_{0}=6.0 \AA$ and $\epsilon_{0}=4.88 \mathrm{kcal} / \mathrm{mol}$ adopted as the LJ parameters of the solute. The parameters for $\mathrm{SPC} / \mathrm{E}$ water are: $\sigma_{\mathrm{LJ}}=3.166 \AA$ and $\epsilon_{\mathrm{LJ}}=0.15535$ $\mathrm{kcal} / \mathrm{mol}^{6}$

We examine the ionic mobility of the solute particle by performing a set of simulations with six different charges: $q=0.01,0.1,0.5,1.0,1.5,2.0$. A cubic simulation box with the length $L=35.2 \AA$ contains $\sim 1500 \mathrm{SPC} / \mathrm{E}$ water molecules and a single charged solute. MD simulations are performed at $300 \mathrm{~K}$ with the periodic boundary conditions and the PPPM Ewald method to compute electrostatic interactions. The cutoff distance for the nonbonded interactions is $14 \AA$. For each solute charge, the system is equilibrated for $10 \mathrm{~ns}$, followed by production runs of $100 \mathrm{~ns}$ in the NVT ensemble with the saving time of 2 ps. To achieve sufficient resolution for the force correlation function, separate simulations with the length of $20 \mathrm{~ns}$ were performed with the saving time of $10 \mathrm{fs}$.

The simulation protocol for azurin mutants was adopted as described elsewhere. ${ }^{7}$ Briefly, the X-ray diffraction structure (resolution $2.70 \AA$ ) of pseudomonas aeruginosa azurin $\left(\mathrm{PaAz}, \mathrm{PDB} 1 \mathrm{AZU}^{8}\right)$ was used as the starting configuration for MD simulations with CHARMM $27^{9}$ force field for the peptide chain. The

\footnotetext{
a) Electronic mail: dmitrym@asu.edu
}

parameters for the copper site in the reduced (Red) and oxidized $(\mathrm{Ox})$ states were adopted by combining atomic charges from Ullmann et $\mathrm{al}^{10}$ with the bonded parameters from Swart et al ${ }^{11-13}$ and van der Waals parameters from Nan-Jie et al. ${ }^{14}$ The mutants were solvated with 36469 TIP3P water molecules resulting in a cubic simulation box with the length $L=104 \AA$ consisting of the total of 111278 atoms.

We simulated 6 charge states of azurine ( $q$ from 0 to $-5)$. Patches were used to change the protonation states of residues. The psfgen plugin of VMD was used to apply the patches. The charge state of $q=-1 e$ was created by replacing the histidine residue (res. No. 83) from the Ox state of the protein with the protonated histidine (HSP). The zero charge state was created by taking the previous system and replacing the glutamate residue (res. No. 104) with the protonated residue (GLUP). The charge state of $q=-4 e$ was created by replacing the lysine residue (res. No. 27) from Red state with the neutral lysine (LSN). Finally, the charge state of $q=-5 e$ was created by taking the $q=-4 e$ system and replacing the lysine residue (res. No. 24) with the neutral lysine (LSN). NAMD software program ${ }^{15}$ was used in MD runs with the time step of $2.0 \mathrm{fs}$. The lengths of simulation trajectories for Ox and Red states were 300 ns. For HSP83, LYS27, and LYS24 mutants the simulations were run for $200 \mathrm{~ns}$, and for GLU104 mutant the simulation length was 100 ns. Same minimization and NPT equilibration procedures were applied to the $\mathrm{Ox}$ and Red states and to all mutants.

Simulations of force correlation functions required smaller integration step of $1 \mathrm{fs}$ and the saving frequency of 5 fs. Those simulations were performed for $143 \mathrm{~ns}$ $(q=0), 211 \mathrm{~ns}(q=-1 e), 301 \mathrm{~ns}(q=-2 e,-3 e)$, and $211 \mathrm{~ns}(q=-4 e,-5 e)$.

\section{SII. ELECTROSTATIC FORCE}

One start by solving the boundary-value problem for the off-center charge inside a spherical sphere immersed in the dielectric with the dielectric constant $\epsilon$. The standard electrostatic problem has the following solution at $r \geq R$

$$
\phi(r)=\sum_{\ell=0}^{\infty} \frac{s^{\ell}}{r^{\ell+1}} \frac{2 \ell+1}{\ell(\epsilon+1)+\epsilon} P_{\ell}(\cos \theta)
$$

where $P_{\ell}(x)$ is the Legendre polynomial. ${ }^{16}$

At each point of the spherical dividing surface we define the normal, $E_{n}=\hat{\mathbf{n}} \cdot \mathbf{E}$, and tangential, $E_{t}=\hat{\boldsymbol{\theta}} \cdot \mathbf{E}$, 


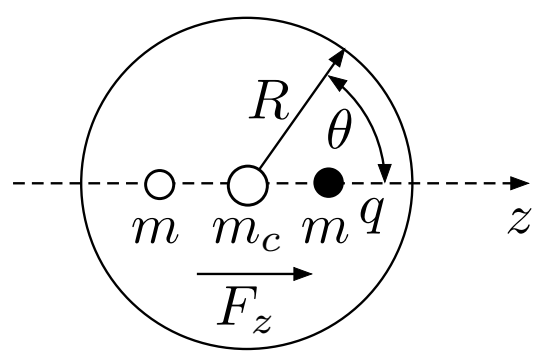

Figure S1. Schematic drawing of the solute with the off-center charge $q$ and three masses: $m$ at distances $R / 2$ from the center and $m_{c}$ at the solute center. The spherical solute has the radius $R$ and interacts with SPC/E water with the LennardJones and electrostatic potentials.

components of the electric field $\mathbf{E}=-\nabla \phi$. These components are given as

$$
\begin{aligned}
E_{n} & =\sum_{\ell=0}^{\infty} \frac{s^{\ell}}{r^{\ell+2}} \frac{(\ell+1)(2 \ell+1)}{\ell(\epsilon+1)+\epsilon} P_{\ell}(\cos \theta), \\
E_{t} & =\sum_{\ell=0}^{\infty} \frac{s^{\ell}}{r^{\ell+2}} \frac{2 \ell+1}{\ell(\epsilon+1)+\epsilon} P_{\ell}^{\prime}(\cos \theta) \sin \theta
\end{aligned}
$$

where $s$ is the distance from the geometric center of the solute to the charge (Figure S1).

These equations are used to calculate the $z$-projection of the dielectric force acting on the solute

$$
F_{z}=\oint T_{z i} \hat{n}_{i} d S
$$

where the Maxwell stress tensor ${ }^{17}$ is a sum of

$$
\sigma_{\alpha \beta}=\frac{\epsilon}{4 \pi}\left[E_{\alpha} E_{\beta}-\frac{1}{2} \delta_{\alpha \beta} E^{2}\right]
$$

and the component arising from the dielectric constant changing with the density $\rho$

$$
T_{\alpha \beta}=\sigma_{\alpha \beta}+\frac{1}{8 \pi} \delta_{\alpha \beta} E^{2} \rho\left(\frac{\partial \epsilon}{\partial \rho}\right)_{T}
$$

The first term in the tensor produces the $z$-projection of the force ${ }^{18}$

$F_{z}^{(1)}=\left(R^{2} / 4\right) \int_{-1}^{1}\left[\left(E_{n}^{2}-E_{t}^{2}\right) \cos \theta-2 E_{n} E_{t} \sin \theta\right] d \cos \theta$

where $\theta$ is the polar angle (Figure S1). Correspondingly, for the second term one gets

$$
F_{z}^{(2)}=\frac{R^{2}}{4} \rho\left(\frac{\partial \epsilon}{\partial \rho}\right)_{T} \int_{-1}^{1}\left(E_{n}^{2}+E_{t}^{2}\right) \cos \theta d \cos \theta
$$

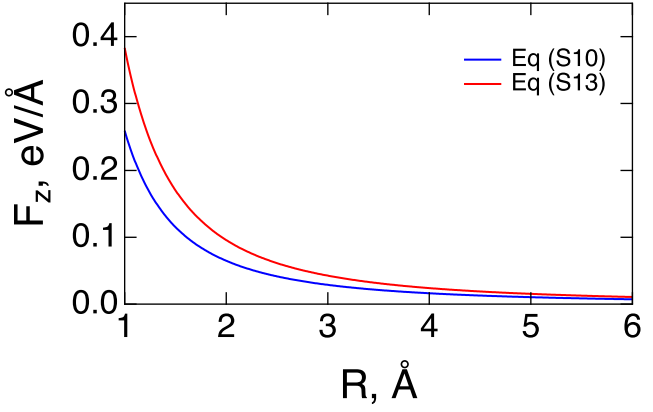

Figure S2. Force $F_{z}$ vs $R$ calculated from Eqs. (S10) and (S13) for the parameters of SPC/E water: $\epsilon=71$ and $\rho(\partial \epsilon / \partial \rho)_{T}=$ $83 ; s=R / 2$.

Calculation of this integral requires a number of integrals involving the Legendre polynomials, which are listed here for convenience

$$
\begin{array}{rl}
\int_{-1}^{-1} & d x x P_{l}(x) P_{m}(x) \\
& =\frac{2(\ell+1)}{(2 \ell+1)(2 \ell+3)} \delta_{m, l+1}+\frac{2 \ell}{(2 \ell-1)(2 \ell+1)} \delta_{m, l-1}, \\
\int_{-1}^{-1} & d x\left(1-x^{2}\right) P_{l}(x) P_{m}^{\prime}(x) \\
= & \frac{2(\ell+1)(\ell+2)}{(2 \ell+1)(2 \ell+3)} \delta_{m, l+1}-\frac{2 \ell(\ell-1)}{(2 \ell-1)(2 \ell+1)} \delta_{m, l-1}, \\
\int_{-1}^{-1} d x & =\frac{2 \ell(\ell+1)(\ell+2)}{(2 \ell+1)(2 \ell+3)} \delta_{m, l+1}+\frac{2 \ell(\ell-1)(\ell+1)}{(2 \ell-1)(2 \ell+1)} \delta_{m, l-1}
\end{array}
$$

From these equations, the force in Eq. (S6) integrates to zero

$$
F_{z}^{(1)}=0
$$

On the other hand, the compression component becomes

$$
\begin{aligned}
F_{z}=F_{z}^{(2)}=\frac{q^{2}}{R^{2}} \rho\left(\frac{\partial \epsilon}{\partial \rho}\right) \sum_{T} \sum_{\ell=0}^{\infty}\left(\frac{s}{R}\right)^{2 \ell+1} \\
(\ell+1) A_{\ell+1}\left[A_{\ell}+A_{\ell+2}\left(\frac{s}{R}\right)^{2}\right]
\end{aligned}
$$

with

$$
A_{\ell}=\frac{\ell+1}{\ell(\epsilon+1)+\epsilon}
$$

The derivation performed here allows both the normal and tangential components of the Maxwell stress at the solute surface. One can anticipate that the liquid might 


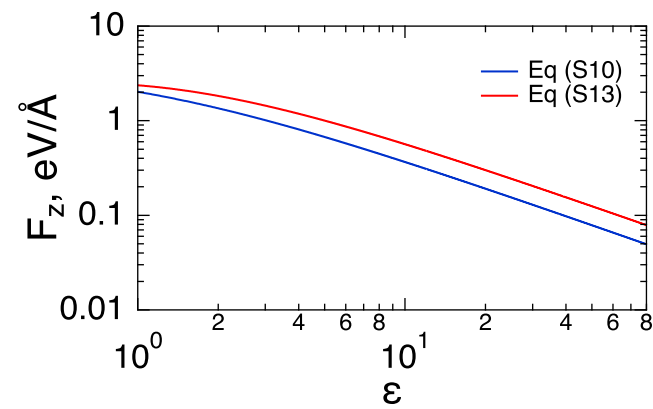

Figure S3. Force $F_{z}$ vs $\epsilon$ calculated from Eqs. (S10) and (S13): $R=2 \AA, s=R / 2, \rho(\partial \epsilon / \partial \rho)_{T}=\epsilon$.

Table S1. Average values of component forces $\left\langle\tilde{F}_{a}\right\rangle, a=$ $\mathrm{E}, \mathrm{vdW}$ for the spherical solute (Figure S1) calculated in the solute body frame $(\mathrm{eV} / \AA)$. The total force is the sum of electrostatic and vdW components.

\begin{tabular}{llll}
\hline \hline$q / e$ & $\mathrm{E}$ & $\mathrm{vdW}$ & Total \\
\hline 0.01 & -0.0004 & -0.013 & -0.014 \\
0.1 & -0.0009 & -0.013 & -0.013 \\
0.5 & 0.070 & -0.091 & -0.020 \\
1.0 & 0.269 & -0.674 & -0.405 \\
1.5 & 0.547 & -1.60 & -1.049 \\
2.0 & 0.981 & -2.82 & -1.837 \\
\hline \hline
\end{tabular}

not sustain the tangential stress and flow instead. In that case, the $z$-axis force becomes

$$
F_{z}=\left(R^{2} / 4\right)\left(\epsilon+\rho\left(\frac{\partial \epsilon}{\partial \rho}\right)_{T}\right) \int_{-1}^{1} d \cos \theta \cos \theta E_{n}^{2}
$$

Similarly with the above, one obtains

$$
F_{z}=\frac{q^{2}}{R^{2}}\left(\epsilon+\rho\left(\frac{\partial \epsilon}{\partial \rho}\right)_{T}\right) \sum_{\ell=0}^{\infty}(\ell+1) A_{\ell} A_{\ell+2}\left(\frac{s}{R}\right)^{2 \ell+1}
$$

The resulting force is about $50 \%$ higher than in Eq. (S10) (Figure S2). We also find that the force $F_{z}$ strongly increases with lowering $\epsilon$ due to the reduction of screening by the polar liquid in the interface (Figure S3).

\section{SIII. ELECTROSTATIC AND VDW FORCES}

The averages and variances of electrostatic and vdW forces acting on the spherical solutes with changing values of $q$ are listed in Tables S1 and S2. The forces are calculated in the solute body frame and the values listed in Table S1 are projections on the symmetry axis of the solute. There is an incomplete compensation between the average electrostatic and vdW forces for the spherical solute. In contrast, the forces calculated in the body frame of the protein produce zero in the average

$$
\left\langle\mathbf{F}_{E}\right\rangle=-\left\langle\mathbf{F}_{\mathrm{vdW}}\right\rangle
$$

Table S2. Variances of component forces $\beta^{2}\left\langle\left(\delta F_{a}\right)^{2}\right\rangle, a=$ $\mathrm{E}, \mathrm{vdW}$ for the spherical solute (Figure S1). Also listed is the cross correlation $\beta^{2}\left\langle\delta \mathbf{F}_{E} \cdot \delta \mathbf{F}_{\mathrm{vdW}}\right\rangle$ and the variance of the total force.

\begin{tabular}{lllll}
\hline \hline$q / e$ & $\mathrm{E}$ & $\mathrm{vdW}$ & Cross & Total \\
\hline 0.01 & 0.002 & 129 & 0.038 & 128.5 \\
0.1 & 0.242 & 136 & 0.010 & 136.3 \\
0.5 & 16.7 & 180 & -24.0 & 149.0 \\
1.0 & 135.6 & 1034 & -332 & 507 \\
1.5 & 580 & 5029 & -1641 & 2326 \\
2.0 & 1878 & 15078 & -5181 & 6593 \\
\hline \hline
\end{tabular}

The direction of the average force $\left\langle\mathbf{F}_{E}\right\rangle$ in the protein body frame is chosen as the symmetry axis to which forces are projected to calculate their variances.

The variance of the total force acting on the spherical solute becomes increasingly anisotropic with increasing $q$ (Table S3). The breadth of fluctuations in the projection perpendicular to the rotation axis $(\perp)$ becomes lower than the corresponding force fluctuations along the rotational axis of the solute. The force relaxation times $\tau_{F}^{\perp, \|}$ also increasingly separate as the hydration shell becomes more tight under the electrostatic pull of the solute charge (Table S3).

The variances of electrostatic and vdW forces acting on the off-center solute show a dependence on the solute charge of the form $a q^{2}+b q^{4}$. This dependence is analyzed for the electrostatic component in Figure $2 d$ in the main text. We follow the same steps for the vdW component here.

According to the general picture of structured solvation shell, the fluctuations of the vdW force can be represented by the term describing the rotations of the shell with the solute (the first summand) and the term representing density-induced fluctuations of vdW force in the body frame (the second summand)

$$
\delta \mathbf{F}_{\mathrm{vdW}}=\left\langle F_{\mathrm{vdW}}^{z}\right\rangle \hat{\mathbf{u}}+\delta \tilde{\mathbf{F}}_{\mathrm{vdW}}
$$

One obtains for the variance

$$
\left\langle\left(\delta F_{\mathrm{vdW}}\right)^{2}\right\rangle=\left\langle F_{\mathrm{vdW}}^{z}\right\rangle^{2}+\left\langle\left(\delta \tilde{F}_{\mathrm{vdW}}\right)^{2}\right\rangle
$$

Since $\left\langle F_{\mathrm{vdW}}^{z}\right\rangle$ scales linearly with $q^{2}$, the first terms is proportional to $q^{4}$. If the second term scales as $\propto q^{2}$, one anticipates that $\left\langle\left(\delta F_{\mathrm{vdW}}\right)^{2}\right\rangle / q^{2}$ should be a linear function of $q^{2}$. This expectation is supported by MD simulations (Figure S4).

In contrast to E and vdW forces for the off-center solute, the variances of the perpendicular and parallel projections of the force acting from water on protein mutants show no substantial dependence on the protein charge.

\section{SIV. TRANSLATIONAL DIFFUSION}

The translational diffusion coefficient of the spherical solute (Figure S1) was calculated from the linear portion 


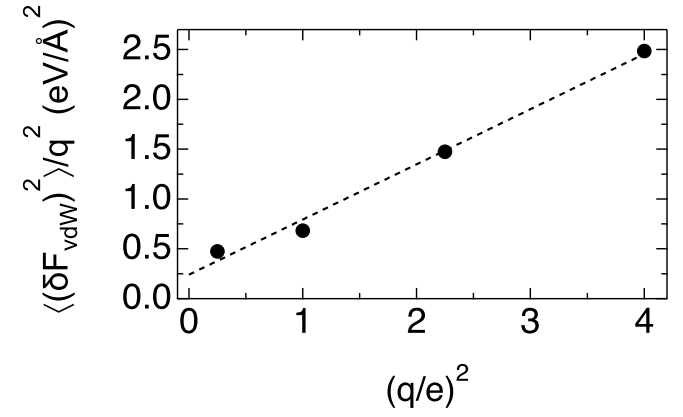

Figure S4. Variance of the vdW force divided by $q^{2}$ (MD, points) vs $q^{2}$. The dashed line is a linear fit through the points.

Table S3. Variance of the total force in the body frame in the parallel $(\|)$ and perpendicular $(\perp)$ projections to the rotation axis $\left((\mathrm{eV} / \AA)^{2}\right)$ and the corresponding force relaxation times $\tau_{F}^{\perp, \|}(\mathrm{ps})$.

\begin{tabular}{lllll}
\hline \hline$q / e$ & $\left\langle\left(\delta \tilde{F}_{\perp}\right)^{2}\right\rangle$ & $\left\langle\left(\delta \tilde{F}_{\|}\right)^{2}\right\rangle$ & $\tau_{F}^{\perp}$ & $\tau_{F}^{\|}$ \\
\hline 0.01 & 0.0563 & 0.02877 & 0.0123 & 0.015 \\
0.1 & 0.0594 & 0.03 & 0.015 & 0.021 \\
0.5 & 0.06235 & 0.03436 & 0.012 & 0.015 \\
1.0 & 0.0846 & 0.0949 & 0.006 & 0.0096 \\
1.5 & 0.14078 & 0.29167 & 0.0023 & 0.012 \\
2.0 & 0.2499 & 0.72569 & 0.0022 & 0.0086 \\
\hline \hline
\end{tabular}

of the time dependence of the mean squared displacement. The velocity time correlation function is oscillating in this case and asserting the contribution of the tail portion of the correlation function to the integral is challenging. The displacement approach is more reliable in this case. In contrast, the velocity correlation function is smoothly decaying for proteins and it was integrated after fitting to a linear superposition of decaying exponents and explained elsewhere. ${ }^{7}$ The result of both calculations where corrected for finite size effects according to the following equation ${ }^{19}$

$$
D_{t}=D_{\mathrm{PBC}}+2.837297 k_{\mathrm{B}} T /(6 \pi \eta L)
$$

where $\eta$ is the shear viscosity of water. The value $\mathrm{e}^{20}$ $\eta=0.722 \mathrm{cP}$ is adopted for $\mathrm{SPC} / \mathrm{E}$ water used in our simulations. The correction substantially affects the reported diffusion constants as is demonstrated in Figure S5.

\section{SV. ROTATIONAL DIFFUSION}

The rotational dynamics in MD simulations is accessed through the time correlation function of the solute unit vector

$$
\Phi_{r}(t)=\langle\hat{\mathbf{u}}(t) \cdot \hat{\mathbf{u}}(0)\rangle
$$

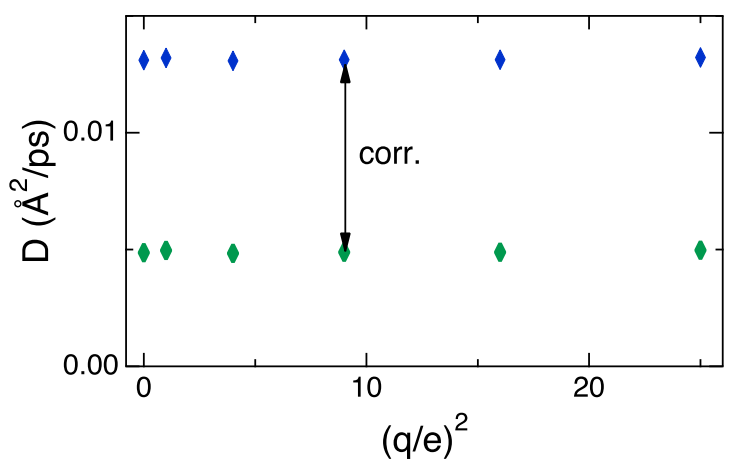

Figure S5. Apparent diffusion constants of protein mutants from MD simulations (green points) and the values corrected according to Eq. (S17) (blue points): "corr." marks the correction term in Eq. (S17).

The relaxation time reported in Figure 2c in the main text is the integral relaxation time

$$
\tau_{r}=\int_{0}^{\infty} d t \Phi_{r}(t)
$$

The rotational correlation function qualitatively changes its behavior with increasing the magnitude of the off-center charge. It decays through oscillations reflecting inertial relaxation of the solute orientations at small $q$ and turns into a smoothly decaying function, which is well fitted by two decaying exponents, at larger values of the off-center charge $q$. The dynamics are thus inertial at small electrostatic interactions becoming overdamped with increasing electrostatic interactions.

The time correlation function from MD was fitted to the following model function allowing oscillations ${ }^{21}$

$$
\Phi_{r}(t)=A e^{-\omega_{g}^{2} t^{2} / 2}+(1-A) e^{-\alpha t}(\cos \omega t+(\alpha / \omega) \sin \omega t)
$$

with the following fitting parameters: $A, \omega_{g}, \omega, \alpha$. The integral relaxation time becomes

$$
\tau_{r}=A \sqrt{\frac{\pi}{2}} \frac{1}{\omega_{g}}+(1-A) \frac{2 \alpha}{\omega^{2}+\alpha^{2}} .
$$

According to Ref. 22, the rotational time calculated from simulations needs to be multiplied with the correction factor $1-v_{m} / V$ accounting for finite-size effects, where $v_{m}$ is the molecular volume and $V=L^{3}$ is the volume of the simulation shell. For the current simulation setup, this factor amounts to 0.997 and can be neglected within the numerical uncertainties of the fitting algorithm.

\section{SVI. FORCE-FORCE CORRELATION FUNCTION}

The force autocorrelation functions for proteins were calculated from trajectories with the integration time of 
Table S4. Variance of the total force in the body frame in the parallel $(\|)$ and perpendicular $(\perp)$ projections to the direction of the dipole moment of azurin mutants $\left((\mathrm{eV} / \AA)^{2}\right)$. Also shown are the force relaxation times $\tau_{F}^{\perp, \|}$ and the relaxation time of the total force $\tau_{F}(\mathrm{ps})$.

\begin{tabular}{llllll}
\hline \hline$q / e$ & $\left\langle\left(\delta \tilde{F}_{\perp}\right)^{2}\right\rangle / 2$ & $\left\langle\left(\delta \tilde{F}_{\|}\right)^{2}\right\rangle$ & $\tau_{F}^{\perp}$ & $\tau_{F}^{\|}$ & $\tau_{F}$ \\
\hline 0 & 6.450 & 6.419 & 0.0057 & 0.0055 & 0.00563 \\
-1 & 6.361 & 6.241 & 0.0055 & 0.0055 & 0.00557 \\
-2 & 6.499 & 6.529 & 0.0070 & 0.0082 & 0.00622 \\
-3 & 6.436 & 6.381 & 0.0068 & 0.0084 & 0.00627 \\
-4 & 6.499 & 6.331 & 0.0066 & 0.0085 & 0.00605 \\
-5 & 6.367 & 6.313 & 0.0060 & 0.0056 & 0.00624 \\
\hline \hline
\end{tabular}

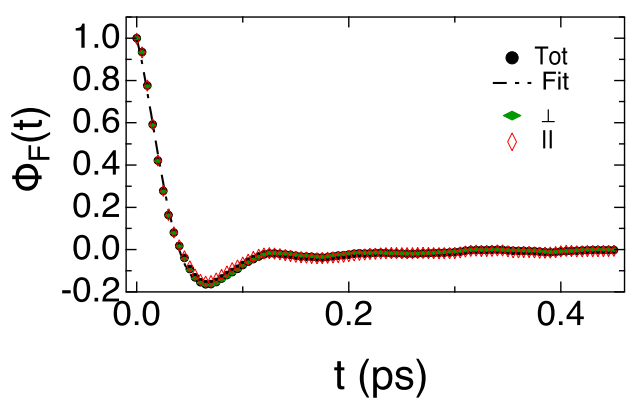

Figure S6. $\Phi_{F}(t)$ for the total correlation function in eq (S22) and the corresponding parallel and perpendicular projections calculated for the reduced state of azurin $(q=-3 e)$. The dashed line is the fit to eq (S23) with unconstrained $A$ and $B$ constants.

$1 \mathrm{fs}$ and the saving time of $5 \mathrm{fs}$. The forces between water and the protein in the laboratory frame of the simulation cell $\mathbf{F}(t)$ were projected to the body frame of the protein to produce $\tilde{\mathbf{F}}(t)$ at each step of the simulation trajectory. The body frame of the protein was constructed from the unit vector of the protein dipole $\left(\hat{\mathbf{e}}_{1}\right)$ and the vector $\hat{\mathbf{e}}_{2}$ perpendicular to $\hat{\mathbf{e}}_{1}$ in the plane formed by the protein dipole and the radius vector connecting the protein center of mass to the position of the $\mathrm{Cu}$ atom in azurin's active site. Finally, the third direction $\hat{\mathbf{e}}_{3}$ is the unit vector perpendicular to the plane formed by $\hat{\mathbf{e}}_{1}$ and $\hat{\mathbf{e}}_{2}$. The set of vectors $\hat{\mathbf{e}}_{i}, i=1,2,3$ was produced for each frame of the simulation trajectory.

The typical force autocorrelation functions for the parallel projection of the force $\tilde{F}_{\|}(t)=\hat{\mathbf{e}}_{1} \cdot \mathbf{F}(t)$ and the perpendicular projection $\tilde{F}_{\perp}(t)=\mathbf{F}(t)-\hat{\mathbf{e}}_{1} F_{\|}(t)$ are compared in Figure S6 to the total time correlation function

$$
C_{F}(t)=\langle\delta \mathbf{F}(t) \cdot \delta \mathbf{F}(0)\rangle
$$

where $\delta \mathbf{F}(t)=\mathbf{F}(t)-\langle\mathbf{F}\rangle$.

All force correlation functions decay on the time scale of 5-6 fs and display oscillations. In most cases the normalized correlation functions $\Phi_{F}(t)=C_{F}(t) / C_{F}(0)$ could be successfully fitted to the following fitting func- tion

$$
\begin{aligned}
\Phi_{F}(t) & =A e^{-\alpha_{1} t}+B e^{-\alpha_{2} t} \sin \left(\omega_{b} t\right) \\
& +(1-A) e^{-\alpha_{2} t}\left(\cos \omega t+\left(\alpha_{2} / \omega\right) \sin \omega t\right)
\end{aligned}
$$

which satisfies the condition $\Phi_{F}(0)=1$. Since the last term satisfies the condition of vanishing derivative at $t=0,{ }^{21}$ one can additionally put $B=\alpha_{1} A / \omega_{b}$ to satisfy $\Phi_{F}^{\prime}(0)=0$. However, this constraint significantly affects the quality of the fit. Given that uncertainties introduced by fitting are hard to access, the force relaxation times of protein mutants shown in Table S4 were obtained by numerical integration of the relaxation functions interpolating between the simulation points in the time interval $0 \leq t \leq 0.5 \mathrm{ps}$. A similar procedure was applied to producing parallel and perpendicular relaxation times for the spherical solutes listed in Table S3. However, we used fitting function given by eq $(\mathrm{S} 23)$ for this set of data since the quality of the fits was far superior.

\section{REFERENCES}

${ }^{1}$ S. Rajamani, T. Ghosh, and S. Garde, J. Chem. Phys. 120, 4457 (2004).

${ }^{2}$ S. Koneshan, J. C. Rasaiah, R. M. Lynden-Bell, and S. H. Lee, J. Phys. Chem. B 102, 4193 (1998).

${ }^{3}$ P. Kumar and S. Yashonath, J. Molec. Liq. 277, 506 (2019).

${ }^{4}$ J. C. Rasaiah and R. M. Lynden-Bell, Phil. Trans. R. Soc. A 359, 1545 (2001).

${ }^{5}$ M. P. Allen and D. J. Tildesley, Computer Simulation of Liquids (Clarendon Press, Oxford, 1996).

${ }^{6}$ H. J. C. Berendsen, J. R. Grigera, and T. P. Straatsma, J. Phys. Chem. 91, 6269 (1987).

${ }^{7}$ S. M. Sarhangi and D. V. Matyushov, J. Phys. Chem. Lett. 11, 10137 (2020).

${ }^{8}$ E. Adman and L. Jensen, Israel J. Chem. 21, 8 (1981). ${ }^{9}$ S. E. Feller and A. D. MacKerell, J. Phys. Chem. B 104, 7510 (2000).

${ }^{10}$ R. T. Ullmann and G. M. Ullmann, J. Phys. Chem. B 115, 10346 (2011).

${ }^{11}$ M. van den Bosch, M. Swart, J. G. Snijders, H. J. Berendsen, A. E. Mark, C. Oostenbrink, W. F. van Gunsteren, and G. W. Canters, ChemBioChem 6, 738 (2005).

${ }^{12}$ M. Swart, Density functional theory applied to copper proteins, Ph.D. thesis, University Library Groningen (2002).

${ }^{13}$ V. Rajapandian, V. Hakkim, and V. Subramanian, J. Phys. Chem. B 114, 8474 (2010).

${ }^{14}$ N.-J. Deng, L. Yan, D. Singh, and P. Cieplak, Biophys. J. 90, 3865 (2006).

${ }^{15}$ J. C. Phillips, R. Braun, W. Wang, J. Gumbart, E. Tajkhorshid, E. Villa, C. Chipot, R. D. Skeel, L. Kale, and K. Schulten, J. Comp. Chem. 26, 1781 (2005).

${ }^{16}$ M. Abramowitz and I. A. Stegun, eds., Handbook of Mathematical Functions (Dover, New York, 1972). 
${ }^{17}$ J. D. Jackson, Classical Electrodynamics (Wiley, New York, 1999).

${ }^{18}$ D. V. Matyushov, Mol. Phys. 112, 2029 (2014).

${ }^{19}$ I. C. Yeh and G. Hummer, J. Phys. Chem. B 108, 15873 (2004).

${ }^{20}$ S. H. Lee and J. Kim, Mol. Phys. 117, 1926 (2019).
${ }^{21}$ U. Balucani and M. Zoppi, Dynamics of the Liquid Phase (Clarendon Press, Oxford, 1994).

${ }^{22}$ M. Linke, J. Köfinger, and G. Hummer, J. Phys. Chem. Lett. 9, 2874 (2018). 